\title{
The Method of Separate Phase Adjusting for Dual- Channel Monopulse Tracking System
}

\author{
Fu'an Sun*, Junchao Li, Sanwen Li and Cheng Li \\ China Satellite Maritime Tracking and Control Department, Jiangyin, Jiangsu, China 214431 \\ ${ }^{*}$ Corresponding author
}

\begin{abstract}
Aimed at the limitation of the phase adjusting method now available for the tracking system, the paper puts forward the method of separate phase adjusting. Based on the full testing of signal conveying character, the phase value of any frequency and any channel configuration can be gained by the method on the condition of rational computing and microwave self-checking. The method is quick and accurate, especially on the emergent phase adjusting with changing of target character and tracking channel.
\end{abstract}

Keywords-tracking channel; group delay; separate phase adjusting

\section{INTRODUCTION}

The tracking receiver of dual-channel monopulse[1] is applied widely in the field of precise tracking and measuring radar, with the advantage of simple structure, high precision and easy to realize. Furthermore, it is the most plan applied in the TT\&C system at present[2]. The phase value and gradient of azimuth and pitching is gained by phase adjusting, with which the tracking receiver demodulates the angle error and tracks automatically [3].

The current phase adjusting method such as facing tower, tracking target and releasing beacon ball, can satisfies the requirement of the TT\&C task on the sea on the condition of explicit target character and stable equipment status. However, with rapid the development of space technology, the frequentness and intensity of the TT\&C task on the sea is higher and higher, technology status and equipment configuration is more and more complicated, and the requirement of emergent TT\&C task is more and more urgent. The phase adjusting of dynamic condition on the sea is harder when the target character is not explicit and tracking channel changes for equipment fault. So we need to find new method of phase adjusting.

\section{Phase Analysis of Tracking Channel}

Figure I. is the representative tracking channel of dualchannel monopulse tracking system. When the antenna feed source receives the target signal, sum signal and difference signal are produced by mould coupler, which pass through waveguide, field-effect amplifier, downconverter, slip ring, medium frequency level regulator, switch array and power distributor. At last, the angle error is demodulated by tracking receiver. The transmission delay of between sum signal and difference signal is not the same in the engineering, so the phase difference between sum signal and difference signal is produced.

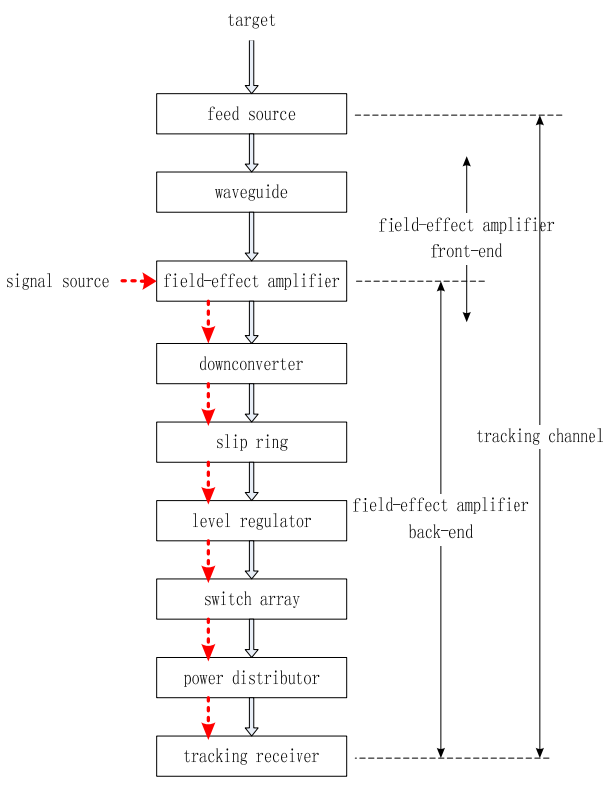

FIGURE I. TRACKING CHANNEL

The transmission delay of between sum signal and difference signal at a certain frequency is fixed, after the tracking channel is debugged. It changes when any device in the tracking channel changes, for which the phase needs to be adjusted again.

The phase adjusting of current method is on the whole tracking channel. As a matter of fact, the capability of feed source and waveguide before field-effect amplifier is very stable, but the capability of device after field-effect amplifier is not so. When any device is fault, the tracking channel needs to be switched according to the require of task.

Microwave self-checking is the regular means for phase checking. The signal produced by signal source conveys directly to the field-effect amplifier, and then passes through the tracking channel to the tracking receiver. The devices between field-effect amplifier and tracking receiver are checked, whose capability is not stable.

The paper puts forward a method of separate phase adjusting, which is quick and accurate taking full advantage of the stability of the devices before field-effect amplifier and 
microwave self-checking covering the devices after field-effect amplifier.

\section{Study ON THE Method of SeParate Phase ADJUSTING}

\section{A. Keystone}

The whole tracking channel is divided into two parts. One is the front-end of the field-effect amplifier, which is from the antenna feed source to the front of the field-effect amplifier, and the other is back-end of the field-effect amplifier, which is from the field-effect amplifier to the tracking receiver. The phase of the whole tracking channel is the sum phase of the front-end and back-end of the field-effect amplifier.

If the one task frequency is $\mathrm{f}_{0}$, e phase of the front-end the field-effect amplifier is $\varphi_{01}$, e phase of the back-end the fieldeffect amplifier is $\varphi_{02}$, $d$ the phase of the whole tracking channel is $\varphi_{0}$. Then

$$
\varphi_{0}=\varphi_{01}+\varphi_{06}
$$

$\varphi_{02}$ can be gained by microwave self-checking, which is accurate and reliable. Then how $\varphi_{01}$ being gained will be discussed.

\section{B. Calculating the Phase of the Front-end the Field-effect Amplifier}

For the stable capability of the front-end the field-effect amplifier, the influence can be ignored.

Group delay is one of key parameter in digital communication system[4].Group delay describes the phase capability of the devices and the delay of signal passing through[5,6].It is only related to f. According to the definition of group delay, if group delay is $\Delta \tau(\mathrm{f})$, then

$$
\Delta \pi(f)=\frac{390(f)}{960} * \frac{1}{f}
$$

If the phase difference between sum signal and difference signal is $A \varphi(f)$, then

$$
\Delta \varphi(f)=\Delta \pi\left(f^{2}\right) * f * 360
$$

According to the formula, the phase of the front-end the field-effect amplifier is only related to $f$. If the function of $\Delta \varphi(f)$ and $f$ is founded, then the phase of the front-end the field-effect amplifier can be calculated at any frequency.

The means of facing tower is adopted to get the relation between the phase of the front-end the field-effect amplifier and frequency. The phase is measured by the high-accuracy phase meter which is connected to the front-end the field-effect amplifier with two same long cable. The range of frequency is $2200 \mathrm{MHz} \sim 2300 \mathrm{MHz}$, whose step is $5 \mathrm{MHz}$. The data are listed in TABLE I.
TABLE I. THE PHASE OF THE FRONT-END THE FIELD-EFFECT AMPLIFIER AT ANY FREQUENCY

\begin{tabular}{l|l} 
frequency $(\mathbf{M H z})$ & the phase (degree) \\
\hline 2200 & 147.50 \\
\hline 2205 & 140.02 \\
\hline 2210 & 139.77 \\
\hline 2215 & 136.60 \\
\hline 2220 & 130.27 \\
\hline 2225 & 129.22 \\
\hline 2230 & 125.00 \\
\hline 2235 & 120.78 \\
\hline 2240 & 117.62 \\
\hline 2245 & 117.27 \\
\hline 2250 & 113.40 \\
\hline 2255 & 110.94 \\
\hline 2260 & 109.94 \\
\hline 2270 & 104.26 \\
\hline 2275 & 103.55 \\
\hline 2280 & 93.36 \\
\hline 2285 & 93.01 \\
\hline 2290 & 85.63 \\
\hline 2295 & 84.22 \\
\hline 2300 & 78.95 \\
\hline
\end{tabular}

Cure fitting of data listed in table 1 on MATLAB can be obtained, which shows the relation between the phase of the front-end the field-effect amplifier and frequency. If the relation is $\mathrm{g}(\mathrm{f})$, then

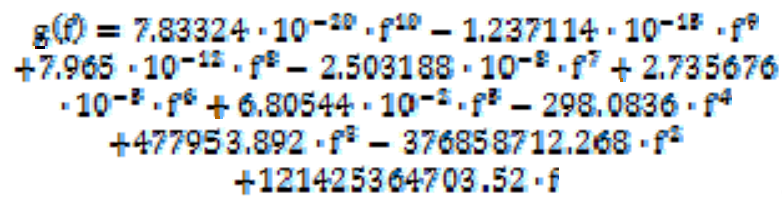

Figure II. is the curve of $g(f)$.

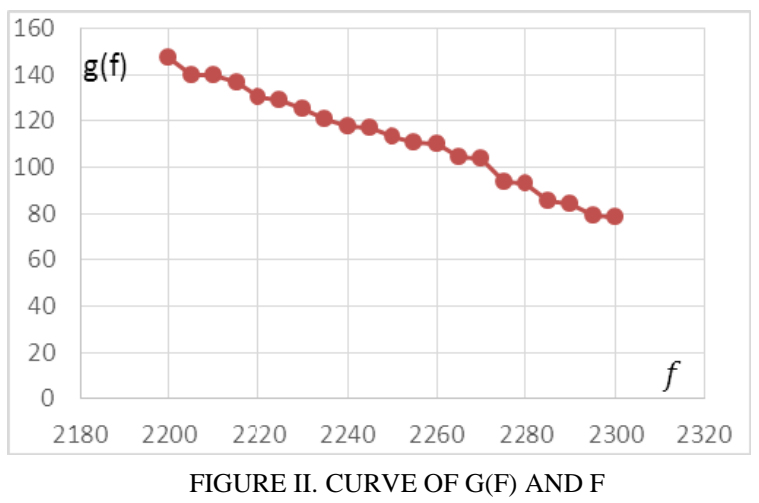

If the task frequency is $f_{0}$, then the phase of the front-end the field-effect amplifier is

$$
\varphi_{01}=g\left(f_{0}\right)
$$

Then the phase of the whole channel is

$$
\varphi_{0}=\varphi_{01}+\varphi_{02}=g\left(f_{0}\right)+\varphi_{08}
$$




\section{Analysis of Application}

On a certain frequency, the phase with the method of separate phase adjusting is compared with other method such as facing tower, tracking target and releasing beacon ball. The data is listed in TABLE II.

\section{TABLE II. THE PHASE WITH DIFFERENT METHOD}

\begin{tabular}{l|l} 
method & phase (degree) \\
\hline facing tower & 145.50 \\
\hline releasing beacon ball & 140.02 \\
\hline tracking target & 139.77 \\
\hline separate phase adjusting & 142.50
\end{tabular}

During the preparation of one, one device did not work. After it is replaced, there is no ready phase for use. Then the phase was obtained by the method of separate phase adjusting, which could fully satisfy the task. So the timeliness and operability of the method is certified.

\section{CONCLUSION}

The paper innovates the method of separate phase adjusting for tracking channel. Based on the full testing of signal conveying character, the phase value of any frequency and any channel configuration can be gained by the method on the condition of rational computing and microwave self-checking. Comparing with other methods, it requires less on exotic environment, cooperation target and tracking channel. It is quick and accurate, especially on the emergent phase adjusting with changing of target character and tracking channel.

\section{REFERENCES}

[1] LIU Jia-xing. Spacecraft TT\&C and Communication Engineering[M].Beijing: National Defense Industry Press, 2010.(in Chinese).

[2] SU Xun, XI Wen-jun. Phase Calibration Technology of Dual-channel Tracking Receiver with Ground Beacon[J].Telecommunication Engineering, 2012, 52(3):268-272. (in Chinese)

[3] WU You-xing. Digital Base Band Technology of Space TT\&C[M].Beijing: National Defense Industry Press, 2010.(in Chinese)

[4] ZHOU Xiao-juan. Methodology Research of Group Delay Measurement[J].Electronic Design Engineering, 2012,20(6):124-126.

[5] Liu Tao. Fast Method of Group Delay Measurement[J].Chinese Journal of Radio Science, 2009,24(2):369-371.

[6] HAYKIN S and VAN VEEN B. Signal and Systems[M]. 2nd ed. New York: Wiley, 2003 